\title{
Téoros
}

Revue de recherche en tourisme

\section{Les camps de vacances}

\section{Serge Choinière et André Lauzon}

Volume 8, numéro 2, juillet 1989

\section{Hébergement et tourisme}

URI : https://id.erudit.org/iderudit/1080322ar

DOI : https://doi.org/10.7202/1080322ar

Aller au sommaire du numéro

\section{Éditeur(s)}

Université du Québec à Montréal

\section{ISSN}

0712-8657 (imprimé)

1923-2705 (numérique)

Découvrir la revue

Citer cet article

Choinière, S. \& Lauzon, A. (1989). Les camps de vacances. Téoros, 8(2), 19-21.

https://doi.org/10.7202/1080322ar d'utilisation que vous pouvez consulter en ligne.

https://apropos.erudit.org/fr/usagers/politique-dutilisation/ 


\section{Serge Choinière* et André Lauzon*}

\section{Les camps de vacances}

Il existe près de 200 camps de vacances au Québec représentant une capacité totale d'hébergement d'environ 25000 lits et la thajorite operent surtout de façon saisonnière. Les camps de vacances constituent un apport non négligeable à l'économie touristique québécoise puisqu'ils génèrent des revenus annuels d'environ 50 millions de dollars.

\section{Les origines}

Formule de vacances plutốt centenaire, lescamps de vacances destines aux jeunes sont apparus au Québec d'abord chez les anglophones, en 1984. alors que le YWCA instituait un camp de vacances à Saint-Adolphe-de-Horward dans les Laurentides. Chez les francophones, ee sont les Grèves de Contrecoeur en Montérégie qui, en 1912, ont fait office de pionniers.

A leur origine, les camps de vacances representaient en quelque sorte un prolongement de l'éducation scolaire, surtout auprès des clientèles francophones. En effet, la majorité des śtablissements étaient gérés par des religicux qui une fois les classes terminées, se retrouvaient en milieu naturel avec d'autres jeunes alors qu'avec le développement industricl et l'urbanisation du début du siècle, le camp de vacanoes devenait un des rares moyens de permettre aux enfants de retoumer à la campagne, l'ẻté venue.

\section{L'Association des Camps du Québec}

La plupart membres de l'Association des Camps du Canada, fondée en 1936, les camps de vacances québecois se sont rencontrés informellement durant plus de vingt ans avant de créer une association provinciale autonothe. C'esten 1961 que fut créé l'Association des Camps du Québec (A.C.Q.).

L'ACQ regroupe la majoritế des camps de vacances du Québec, c'est-ä-dire 120 établissements, et constitue pour le public une forme de garantie de qualité de service. Tous les camps membres de lassociation sont régis par un ensemble de nomes et standards. A titre d'exemples d'exigences de l'ACQ, notons la couverture obligatoire du camps de vacances par une police d'assurances adequate, la qualité d'intervention garantic par des ratios animateurs/enfants, des programmes de sécurité et la confection de fiches d'informations relatives dì la santé et autres détails concemant les enfants, l'entretien du materiel et la sécurité des activités, la salubrité, la qualité et l'équilibre des menus approuvés par wne diététicienne. Afin d'assurer le respect de ces normes, chaque campest soumis à une visite d'inspection a tous les deux ans.

L'Association des Camps du Québec voit aussi a la formation de ses membres par la tenue, à tous les ans, d'un colloque où ceux-ci peuvent y entendre des conferenciers de marque et participer à des ateliers sur divers sujets touchant la gestion des établissements. Les gestionnaires et les administrateurs des camps peuvent également parfaire leur formation en assistant à des stages de formation conçus expressement pour eux alors que les formateurs, cest-à-dire les personnes responsables du personnel d'animation, suivent des stages portant sur leblaboration de programmes et la gestion du personnel.

\section{Communication-marketing}

Cest surtout sous la forme coopérative que se traduisent les activités de communication-marketing destinécs à faire connaitte aux consommateurs les services offerts par les camps de vacances. Chaque camp publie un depliant et plusieurs d'entre eux effectuent des envois postaux à leurs anciens clients mais c'est l'Annuaire des camps de vacances, publié par l'ACQ avec laide du ministère du Loisir, de la Chasse et de la Peche (M.L.C.P.), qui constituerait l'outil principal de communication.

Regroupant l"information pour l'ensemble des établissements, l'Annuaire des camps de vacances de l"ACQ présente un répertoire fort bien documenté que les parents peuvent obtenir dans divers endroits, entre autre dans les CLSC partout au Québec. On trouve dans cet annuaire la liste des camps répertoriés suivant leur spécialisation et leur situation géographique. Certains camps se caractérisent par des activités spécifiques telles par exemple l'équitation, la formation musicale ou l'immersion en langue seconde allors que dautres se specialisent dans laccueil declientëles souffrant d'handicaps physiques ou intellectuels. Les parents trouvent donc à l'intérieur du répertoire le camp de vacances correspondant à leurs attentes ainsi quat celles de leurs enfants.

Des cahiers spéciaux dans les grands quotidiens du Québec, la tenue annuelle de foires des camps de vacances an Complexe Desjardins à Montréal et à la Place Laurier à Québec ainsi qu'une
* Messieurs Serge Choiniere et André Lauzon sont chez Gescona, firme conseils specialisee en hotellerie, en tourisme et en loisir. 
série d'entrevues et autres activités médiatiques completent le plan de promotion de l'Association des camps du Québec. Certains camps de vacances plus en moyen ont également recours à des annonces individuelles dans les médias à des moments stratégiques durant l'année.

\section{La clientèle}

Mais qui fréquentent les camps de vacances? Selon une récente étude réaliséce en 1988 par la firme BMA Marketing-Communication pour le compte du ministere du Loisir, de la Chasge et de la Pêche aupres de quelques 4000 ménages québecois, les clients des camps de vacances sont des enfants ägés en moyenne de 11 ans, provenant d'une famille comptant généralement 2 enfants dont les parents démontrent un niveau de scolarité assez élevé el gagnent pour la plupart des revenus familiaux de plus de 350008 par année.

Toujours selon cette étude, l'achalandage annuel des camps de vacances est estime a un peu plus de 2,2 millions de jours/activites pour des revenus globaux de l'ordre de 50 millions de dollars. Loin d'être en baisse, la fréquentation sest accrue de $32 \%$ au cours des trois demières années, ce qui est fort encourageant dans le contexte actucl d'une sociétế où il $\mathrm{y}$ a moins d'enfants qu'autrefois. Ce phénomène s'explique probablement, selon nous, par le fait que les gens sont plus scolarisés, disposent de plus de moyens et considerent que le camp de vacances est un apport important dans l'education et lat socialisation de l'enfant. Et si l'on tient compte que seulement $56 \%$ des familles ont déjà envoyé un de leurs enfants en camp, il y a encore une bonne part du marché primaire à toucher.

Lescamps de vacances jouissent d'une notoriété enviable auprès des familles québécoises avec enfants et le taux de satisfaction dépasse le $90 \%$. Au nivesu du contenu, il est intéressant de constater que la programmation offerte par le camp est de plus en plus un critère de sélection. Alors qu'en 1983, suivant une étude réalisee d l'époque, la programmation était au $4 \mathrm{c}$ rang dars les lacteurs influençant le choix du camp, elle se situe en 1988 au ler rang. En contrepartie, le prix est moins important et vient au se rang dans les facteurs de choix, comme quoi ce sont la qualité des activités offertes et le bien-être de l'enfant qui priment. Il est d'ailleur's significatif que du début de vacances familiales, le camp de vacances en accapare $27 \%$.

\section{Élargissement de la clientèle et étalement de l'opération}

Mais les camps ne se limitent pas exclusivement a accueillir des enfants en séjour de vacances en période estivale, Depuis la dernière décennie, ils ont ouvert leurs portes à d'autres clientèles en vue d'étaler leur période d'opération. La seconde clientèle en importanec est celle des clas-

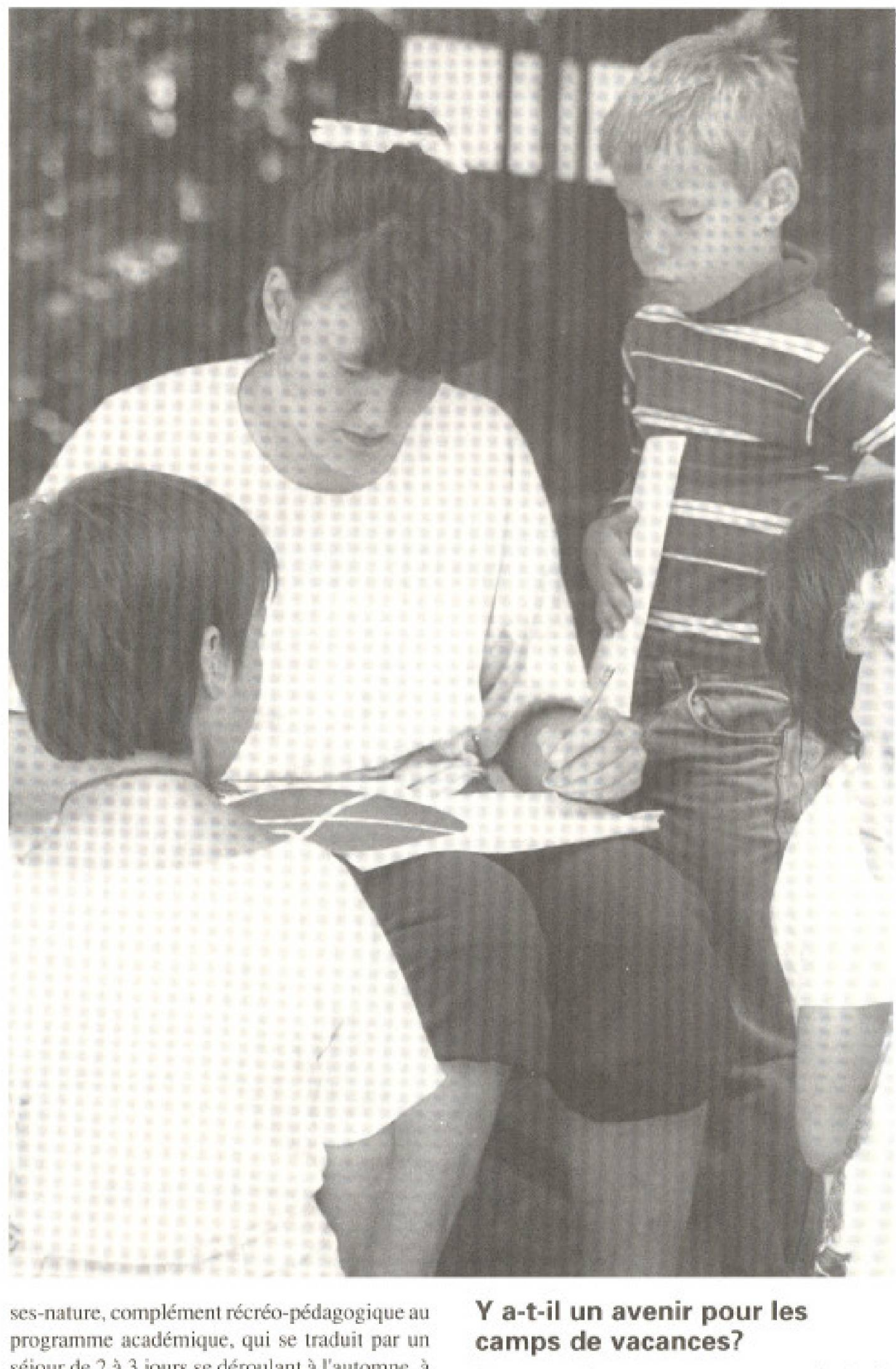

séjour de 2 à 3 jours se déroulant a l'automne, à l'hiver ou encore au printemps. L'Association des Camps du Québec a dailleurs réalisé, en collaboration avee le MLCP, une importante campagne de sensibilisation à la classe-nature avec Claire Pimpare comme porte-parole principale.

Plusieurs camps accueillent également des familles et certains se sont spécialisés dans laccueil de groupes du troisieme âge. Ces demiers, comme d'ailleurs des groupes associatifs en réunions de travatil ou des scouts en camp de week-end, trouvent dans la formule des camps de vacances un mode d'hébergement adéquat et surtout à la mesure de leurs budgets.
Les camps de vacances seront soumis à plusieurs defis de taille sur le chemin de l'an 2000. Plusieurs des equipements sont vieillots et les gestionnaires devront procéder à des rénovations au niveau des bâtiments. En même temps, ils devront adapter leurs equipements afin de répondre aux besoins futurs de la clientèle. Celleci est de plus en plus séloctive et comme les parents sont exigeants dans le choix d'une garderie ou d'une ecole pour leurs enfants, ils proce dent de la même façon pour retenir un camp d'été et prennent la peine deffectuer le magasinage nécessaire.

Les camps de vacances doivent donc se mettre à l'écoute des besoins de la clientèle afin de con- 

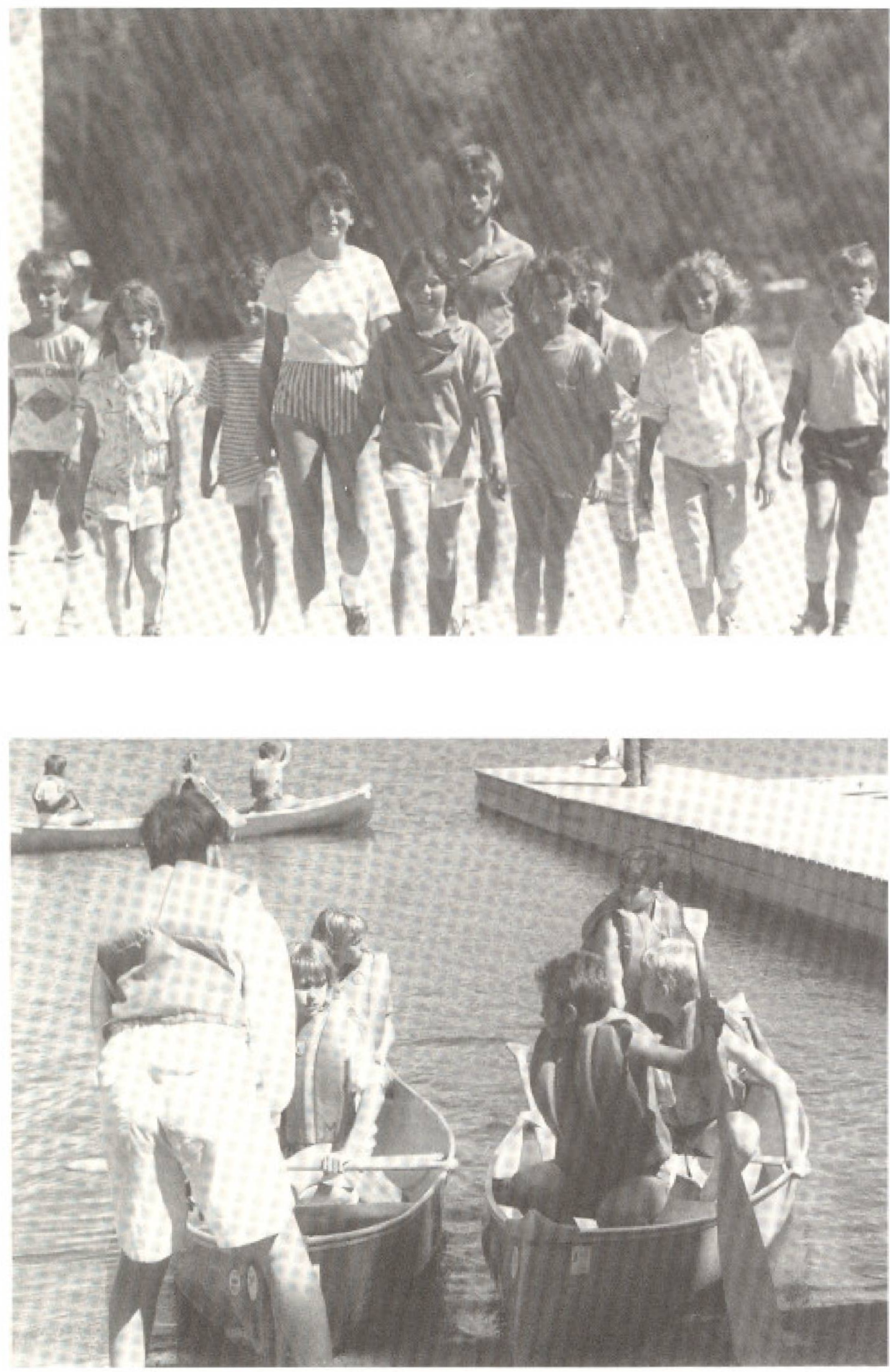

cevoir le produit répondant le plus exactement aux besoins de celle-ci. En ce sens, ils ne different nullement du processus de stratégie de marketing auquel est soumis tout pourvoyeur de service ou fabricant de produit. Ces besoins à combler se situent principalement au niveau du type et de la qualité des activités offertes, le plein air en étant la figure de proue, mais aussi au niveau de la compétence des animateurs qui encadrent ces activités.

Selon les informations qu'il nous a été possible de recueillir, le nombre de camps ou le nombre de places ne connaissent pas une évolution parallèle à l'augmentation de la fréquentation. Selon notre point de vue, le nombre d'établissements devrait sensiblement demeurer le même et la transformation des ćquipements, tel par exemple la modification des dortoirs en aménageant des espaces permettant une plus grande intimité. se traduirait par une capacité d'accueil légèrement moindre mais quand même suffisante pour le marché.

Au niveau de la clientèle primaire, les camps de vacances devront être en mesure d'accueillir les enfants nés de parents néo-québécois qui constitueront, au ry thme où vont les choses, une large part de la population québécoise du 21 e siècle. Ils devront surtout faire en sorte de donner le goût à ces parents d'envoyer leurs enfants en camps de vacances. L'Association des Camps du Quebec est à ce chapitre de plus en plus sollicitée pour des rencontres d'information aupres de divers groupes cthniques. L'ouverture amorcéc vers d"autres clientèles devra se poursuivre de façon encore plus marquéce. Dans le document de stratégie de mise en marché préparee par BMA Marketing-Communication, on invite d'ailleurs les camps a orienter davantage leurs actions de publicité-promotion en ce sens.

Finalement, face à une demande de plus en plus sophistiquéce dans une ère de retrait progressif du soutien financier gouvernemental, les camps de vacances, a l'instar de plusieurs autres équi= pements sociaux de tourisme, évoluent progressivement vers une vision d'entreprise, Cel esprit d'entrepreneurship se traduit chez les gestionnaires de camps surtout par l'attitude face au mode de gestion, au choix des membres du conseild'administration ou dans lapproche pour la réalisation d'une levé de fonds (en oe qui a trait aux camps a but non lucratif). Cest donc la capacité d"adaptation aux nouveaux besoins et la qualité de leur entrepreneurship qui permettront ou non aux camps de vacances de franchir avec succès le cap du tournant du siècle. 